\title{
Active Compensating Method of Sensitivity Temperature Drift of Convection-Based Tilt Sensor
}

\author{
Zheng Yong-hong \\ College of Science \\ Beijing Information Sci. and Tech. University \\ Beijing, China \\ E-mail: hankzyh@126.com
}

\author{
Peng Shi-liu \\ Lab. Of High Temperature and Aerodynamics \\ Institute of Mechanics, CAS \\ Beijing, China \\ E-mail: slpeng@hotmail.com
}

\begin{abstract}
- when environment temperature is changed, the properties of convection-based tilt sensors are changed, which is so-called sensitivity temperature drift. Methods of sensitivity compensation are introduced according to mechanism of this kind of tilt sensor: temperature distribution induced by the heat source plays a key role in sensing tilt angle. The sensitivity temperature drift was researched, a new method of sensitivity temperature active compensating was proposed, and sensitivity temperature active compensating was accomplished.
\end{abstract}

Keywords-convection-based;

temperature

distribution; sensitivity; active compensation

\section{INTRODUCTION}

Convection-based tilt sensors are based on pendulum characteristics of natural convection in a hermetic chamber. Because the mass of gas is small and there is no proof mass, the gas pendulum tilt sensor has large-scale applications for its characteristics of resisting oscillation and shock. At present, the gas pendulum tilt sensors are applied to tank fire control, robot state system, artificial satellite reception system and radar control system etc [1-4].

However, when environment temperature is changed, the properties of convection-based tilt sensor are changed, that is so-called sensitivity drifted with temperature. Although almost all sensors have the problem of sensitivity temperature drift, it is poorer for the convection-based tilt sensors, which caused the range of application limited.

In order to solve this question, a new method of sensitivity temperature active compensating was proposed. According to mechanism of tilt sensor: temperature distribution induced by the heat source plays a key role in sensing tilt, the compensating method of sensitivity temperature drift was researched, and sensitivity temperature active compensation was accomplished.

\section{SENSING MECHANISM OF CONVECTION-BASED}

\section{TILT SENSORS}

Gas pendulum tilt sensor is made according to pendulum characteristic of the natural convection of gas. Because the pendulum of gas pendulum tilt sensor is gas with small mass, it can resist powerful vibration and strong shocks, and can be applied to pose control of ship, tank, radar, and other arms equipments system $[1,2]$.

Sensitive mechanism of gas pendulum tilt sensor is shown in Figure 1. A heat source and two hotwires are placed in a hermetic chamber; a wire like heat source is placed along axis of the chamber, hotwires are placed symmetrically and compose the arms of bridge circuit. When the sensor is in horizontal state two hotwires sense the same temperature of fluid flow, their resistances are equal. The output of bridge circuit is zero. When sensor is in tilt state two hotwires sense the different temperature of the flow, the corresponding resistances are not equal to each other. The bridge outputs a voltage signal corresponding to the angle [5, $6]$.

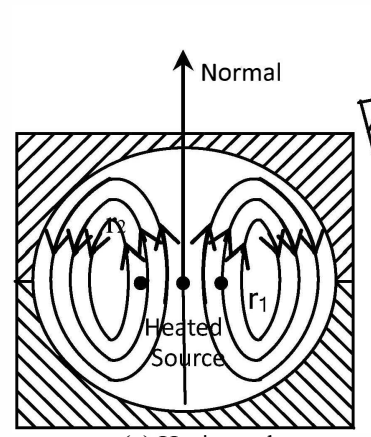

(a) Horizontal state

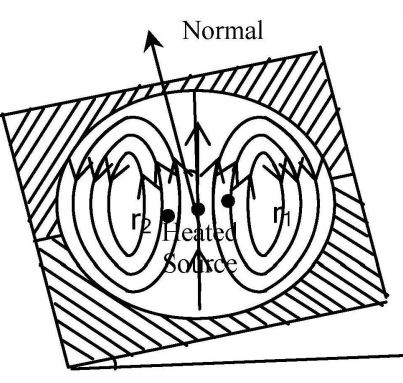

(b) Tilt state
Figure 1. Schematic diagram of gas pendulum

\section{COMPENSATION OF SENSITIVITY}

Sensitivity of convection-based tilt sensors is defined as $\mathrm{d} V / \mathrm{d} \theta$, where $\mathrm{d} V$ is output voltage signal when tilt angle $\mathrm{d} \theta$ is changed. $\mathrm{d} V$ depended on the temperature different of two hot wires under the different tilt and coefficient of voltage switch. However, the experiment results showed that the sensitivity is diversified with different environmental temperatures, this is so-called sensitivity temperature drift.

To resolve the problem of the sensitivity temperature drift, there are two methods at present. One is compensation of hardware, and the other is compensation of software.

\section{A. Compensation of Hardware}

The traditional compensating method of sensitivity provides constant current or constant voltage source, but it is not suitable for reducing the sensitivity temperature drift of convection-based tilt sensor. According to analysis of theory and test, a sensitivity property of sensor is approximate linearity while high order is neglected: 


$$
K\left(T_{a}\right)=K_{0}\left[1+\beta\left(T_{a}-T_{0}\right)\right]
$$

Where, $\mathrm{Ta}$ is environmental temperature, $\mathrm{K} 0$ is sensitivity with $T_{0}$.

Based on formula (1), compensating sensitivity circuit is designed as Fig. 2.

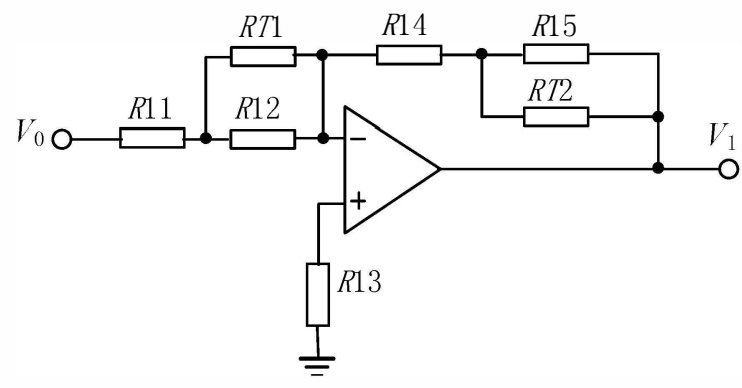

Figure 2. Compensating sensitivity circuit

$R 11, R 12, R 14$, and $R 15$ are resistances. $R T 1$ is resistance with negative temperature-sensitive ratio; $R T 2$ is resistance with positive temperature-sensitive ratio in Fig.2. Magnification factor of circuit can be written as:

$$
A\left(T_{a}\right)=\frac{R 14+R 15 / / R T 2}{R 11+R 12 / / R T 1}
$$

Adjusting $R 11, R 12, R 14$, and $R 15$, letting $A\left(T_{a}\right)$ approach reciprocal of $1+\beta\left(T_{a}-T_{0}\right)$, the output of voltage $V_{1} \approx K_{0} \theta$. By adjusting the above resistances, the sensitivity temperature drift is reduced.

Compensation of hardware has a short responding time, but the consistency of compensate effecting is not better. This method works well for the compensation of linearity error, but it is not good for the compensation of nonlinear error.

\section{B. Compensation of Software}

A built-in system (single chip) is used in compensation of software, which utilizes the conversion of analogy/digit (A/D) to convert an analogy signal of output voltage to a digit signal, processes digit compensation and filtration, and makes digit/analogy (D/A) conversion with useful signal at last. Due to the convenience of digit signal processing, this method is applied to linearity or symmetry compensation. Compensation of software chart is shown on Fig.3, where SCM is a single chip.

The compensation of software has a relative long response time because of basic frequency limit of single chip, it is difficult for high response time system.

It is obvious that both compensation of software and compensation of hardware are passive compensation, which is based on the rule of drift emerged. This means that both above described compensation methods work in order to modify the drift when temperature drift appears. Can a active compensation method be used for this purpose? The following description is a trial.

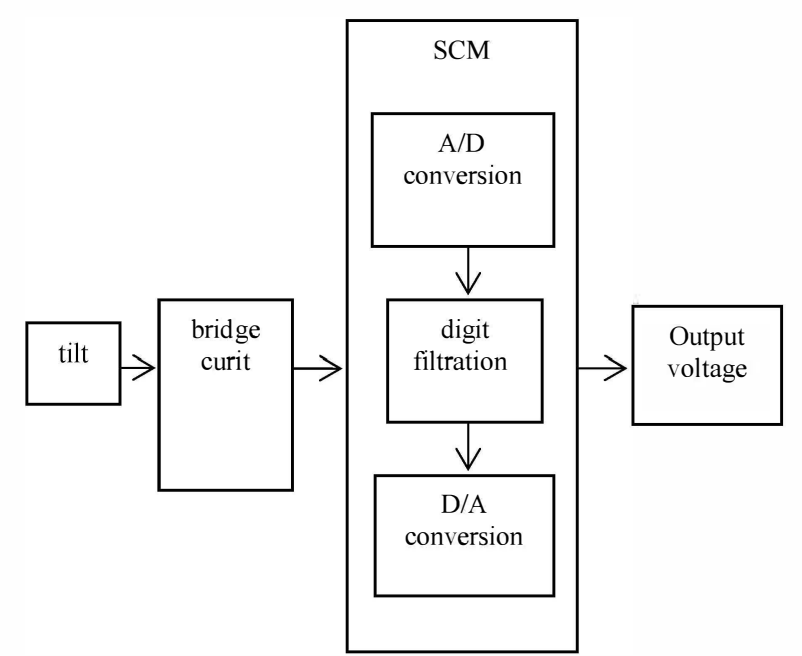

Figure 3. Compensation of software chart

\section{ACTIVE COMPENSATION OF SENSITIVITY}

According to mechanism of tilt sensor, dot heat source is the cause for construction of temperature field and velocity field, which both bring affection to sensing tilt. But the temperature distribution induced by the heat source plays a key role in the sensing tilt [7]. In fact, when environment temperature is changed, the difference between heat source and environment temperature is changed, the properties of convection-based tilt sensors are changed, which caused the sensitivity drift with temperature. It is convenient to use software for analysis in details, and here the software Ansys is used for numerical simulation.

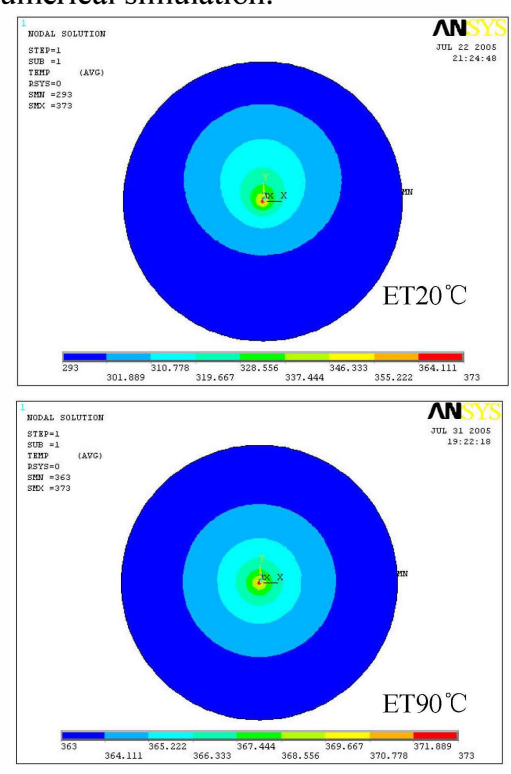

Figure 4. Temperature field diagram

Figure. 4 show temperature distributions with temperature of heat source is $100^{\circ} \mathrm{C}$, environmental temperatures (ET) are $20^{\circ} \mathrm{C}$ and $90^{\circ} \mathrm{C}$. 
From Fig.4, it is very clear that these two temperature fields are different. The output voltages are measured at different tilts, the result are shown in Fig.5. When environment temperature is low, the output voltage is big; when environment temperature is high, the output voltage is small. The lower the environment temperature is, the larger the difference of temperature between the heat source and the environment is, so the gradient of temperature field is biger; the higher the environment temperature is, the smaller the difference of temperature between heat source and environment is, the gradient of temperature field is less.

If temperature field would be kept invariant, sensitivity of sensor is not changed under different environment temperature, sensitivity temperature compensating would be accomplished, which is the essence of active compensation method.

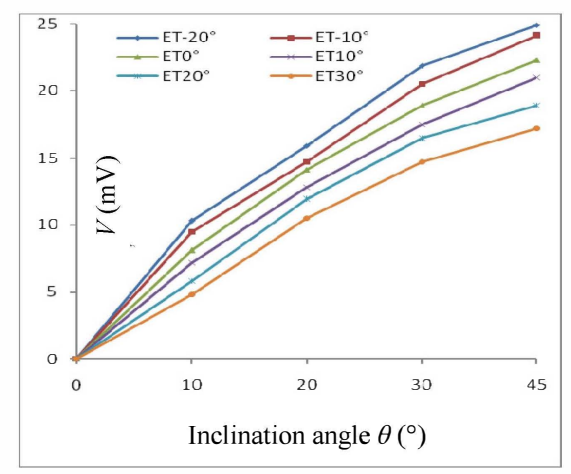

Figure 5. Sensitivity temperature drift at different ET

For temperature distribution induced by the heat source, it is the difference of temperature between heat source and environment that forms temperature field. While keeping the difference of temperature between heat source and environment constant, the gradient of temperature field is fixed[8]. For this purpose, a curit is designed to accomplish active compensation of sensitivity. Fig.6 is simplified compensation curit, in which temperature-sensitive resistance can be calculated based on particular bridge curit. Certainly temperature-sensitive resistance must be a negtive temperature-sensitive resistance(NTC).

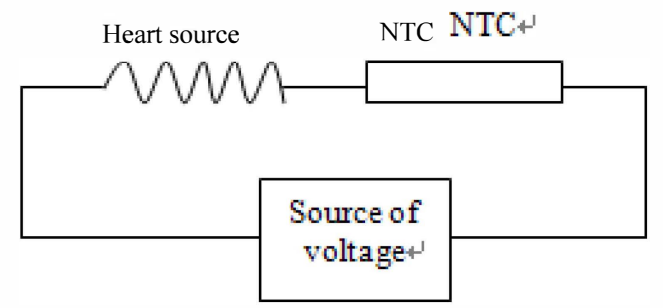

Figure 6. Curit of compensation of sensitivity

\section{CONSEQUENCES}

On the basis of mechanism of the convection-based tilt sensors, the gradient of temperature is kept constant, active compensation of sensitivity is accomplished. The results showed that the sensitivity of convection-based tilt sensors is variety with different environment temperature without the compensation curit. By using the designed curit, the sensitivity of convection-based tilt sensors is basically steadiness at different environment. Activing compensation of sensitivity is finished at last. In Fig.7, (a), (b) and (c) respectively inclination angles are $10^{\circ}, 30^{\circ}$, and $45^{\circ}$. Before compensation (BC), sensitivity changed by a wide margin at different ET. After compensation (AC), sensitivity (SE) is basically invariant at different ET. It is evident that this method is effective.

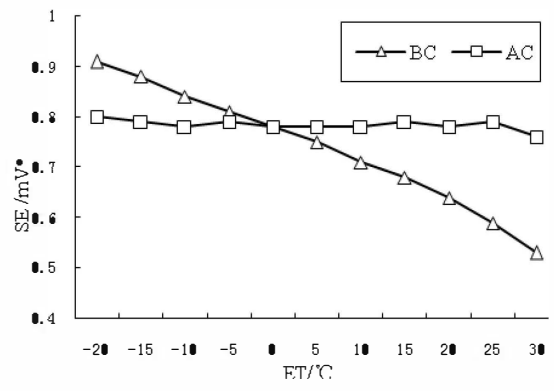

(a) Inclination angle is $10^{\circ}$

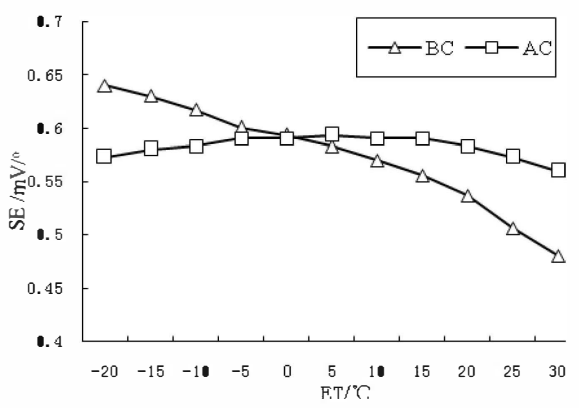

(b) Inclination angle is $30^{\circ}$

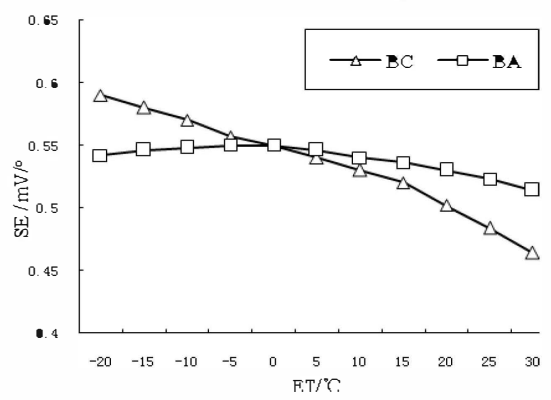

(c) Inclination angle is $45^{\circ}$

Figure 7. Compensation of sensitivity

\section{REFERENCES}

[1] Fu-xue Zhang. "Gas Pendulum Acceleration Sensor," Chinese Journal of Electronics, 2000, 9(1): 14-16

[2] DAUDERSTADT U A, SARRO P M,FRENCH P J. "Temperature Dependence And Drift of a Thermal Accelerometer"J, Sens,Actuators, 1998, A66:244-249. 
[3] DAO R, MORGAN D E, KRIES H H, et al. "Convective Accelerometer And Inclinometer": US, 5581034[P].1996.

[4] Fuxue Zhang, Zhanxian Chen, Yuhua Luo. "Gas Pendulum Tilt Sensor," China CN1064351[P].1992.

[5] F.X Zhang., "Pendulum characteristics of gas in hermetic chamber," Chinese Journal of Electronics, vol.27 (11), 1999, pp.141-142
[6] F.X Zhang., "Pendulum characteristics of natural convection and its application in sensor," Chinese engineering science, 4(8), 2002, pp.50-53.

[7] Yonghong Zheng., "Analysis of stream field of Gas Pendulum Tilt Sensor," Micronanoelectronic Technology, 2007, 7/8 242-244.

[8] Yonghong Zheng., Pengshi Liu. "Application of Flow Similarity to Convection-based Tilt Sensor," Journal of University of Electironic Science and Technology of China. 2007,36 (5) :931-934. 